\title{
Acute effects of ingesting glucose solutions on blood and plasma volume
}

\author{
Gethin H. Evans, Susan M. Shirreffs* and Ronald J. Maughan \\ School of Sport and Exercise Sciences, Loughborough University, Loughborough, Leicestershire LE11 $3 T U$, UK \\ (Received 10 January 2008 - Revised 25 July 2008 - Accepted 20 August 2008 - First published online 8 October 2008)
}

The change in blood and plasma volume following ingestion of glucose solutions of varying concentrations was estimated in twelve healthy male volunteers. Subjects consumed, within a $5 \mathrm{~min}$ period, $600 \mathrm{ml}$ of a solution containing $0,2,5$ or $10 \%$ glucose with osmolalities of 0 (SD 0 ), 111 (SD 1), 266 (SD 7) and 565 (SD 5) mOsm/kg, respectively. Blood samples were collected over the course of $1 \mathrm{~h}$ after ingestion at intervals of $10 \mathrm{~min}$. After ingestion of the $2 \%$ glucose solution, plasma volume increased from baseline levels at 20 min. Plasma volume decreased from baseline levels at 10 and 60 min after ingestion of the $10 \%$ glucose solution. Heart rate was elevated at 10 and 60 min after ingestion of the $10 \%$ glucose solution and decreased at 30 and $40 \mathrm{~min}$ after ingestion of the $2 \%$ glucose solution relative to the average heart rate recorded before drinking. It is concluded that ingestion of hypertonic, energy-dense glucose solutions results in a decrease in plasma and extracellular fluid volume, most likely due to the net secretion of water into the intestinal lumen.

Plasma volume: Extracellular fluid volume: Solution osmolality: Intestinal absorption

The osmolality of an ingested solution determines the osmotic gradient that is the driving force behind the movement of water across the intestinal wall and, therefore, is an important factor determining the direction and rate of water flux in the small intestine. Both rat and human models have suggested that intestinal water absorption is influenced by luminal osmolality, but the relationship will depend on the nature of the added solute. Shi et al. ${ }^{(1)}$ demonstrated that perfusion of a hypotonic carbohydrate solution with an osmolality of $186 \mathrm{mOsm} / \mathrm{kg}$ resulted in a $17 \%$ increase in the rate of water absorption relative to a hypertonic $(403 \mathrm{mOsm} / \mathrm{kg})$ carbohydrate solution. Similarly, Gisolfi et al. ${ }^{(2)}$ reported that a hypertonic $8 \%$ carbohydrate solution (osmolality approximately $460 \mathrm{mOsm} / \mathrm{kg}$ ) containing glucose resulted in reduced net water absorption relative to a hypotonic solution (osmolality approximately $270 \mathrm{mOsm} / \mathrm{kg}$ ). These findings are supported by other segmental perfusion studies ${ }^{(3,4)}$. Perfusion of hypertonic solutions can result in net secretion of water into the lumen. Leiper \& Maughan ${ }^{(5)}$ reported that perfusion of a hypertonic solution, with an osmolality of 488 (SD 53) $\mathrm{mOsm} / \mathrm{kg}$ after transit through the $15 \mathrm{~cm}$ mixing segment of a multilumen tube, resulted in net efflux of water and electrolytes into the lumen over the $30 \mathrm{~cm}$ test segment of the intestine, whereas perfusion of an isotonic glucose-electrolyte solution promoted water and electrolyte uptake. Most intestinal absorption studies have assessed the net rate of water absorption using segmental perfusion techniques. Lambert et al. ${ }^{(6)}$ showed that it is possible to investigate the intestinal absorption characteristics of an orally ingested solution if gastric emptying rate is kept relatively constant. However, investigations that directly perfuse the intestine may not accurately represent fluid absorption characteristics of ingested solutions due to changes in the composition of the solution while in the stomach. Nonetheless, the results of intestinal perfusion studies that have observed a faster rate of fluid absorption following ingestion of hypotonic solutions relative to hypertonic solutions are supported by the results of studies that have used ${ }^{2} \mathrm{H}$-labelled water as a marker of water uptake following ingestion of labelled drinks ${ }^{(7,8)}$.

The rate of intestinal water absorption is especially important when there is a need for rapid replacement of water such as in diarrhoeal disease or when there is a need to match high rates of sweat loss. The stimulation of water absorption by the addition of small amounts of sodium salts and carbohydrate to an ingested solution is the basis of oral rehydration therapy ${ }^{(9-11)}$. The rate of intestinal water absorption is enhanced in the presence of carbohydrate or sodium due to the active co-transport of solute from the intestinal lumen into the mucosa ${ }^{(2,9)}$. Present advice suggests that hypotonic carbohydrate-electrolyte solutions should be consumed when there is a need for rapid fluid replacement, as this results in the most favourable rate of fluid absorption ${ }^{(11,12)}$. Ingestion of hypotonic carbohydrate solutions with an osmolality between about 200 and $260 \mathrm{mOsm} / \mathrm{kg}$ is likely to result in the most favourable rate of water absorption following delivery to the small intestine ${ }^{(8)}$. However, fast rates of fluid absorption can lead to large increases in plasma volume ${ }^{(1)}$, so ingestion of large volumes of hypotonic carbohydrateelectrolyte solutions may stimulate a diuretic response that would be undesirable for maintaining whole-body fluid balance.

Fowkes et al. ${ }^{(13)}$ observed a significant relationship between blood viscosity and blood pressure in individuals aged 55-74 years, and other investigations have also reported 
this relationship ${ }^{(14)}$. Any large reduction in blood or plasma volume following ingestion of hypertonic solutions may lead to an increase in blood viscosity. Because of this, individuals suffering from cardiac insufficiency or hypertension should perhaps avoid sudden or large changes in blood volume that may threaten cardiovascular stability.

The aim of the present study was to estimate blood and plasma volume changes after the ingestion of solutions that differed only in their glucose concentration and, therefore, osmolality.

\section{Methods}

Twelve healthy male subjects (age 25 (SD 5) years, height 176 (SD 5) $\mathrm{cm}$ and body mass 75.3 (SD 12.5) $\mathrm{kg}$ ) volunteered to take part in the present investigation, which had prior approval from the Loughborough University Ethical Advisory Committee (reference number R04/P68). All subjects provided written informed consent and completed a medical screening questionnaire to ensure that they were free from any gastrointestinal problems before starting the study.

The subjects were familiarised with the study protocol before beginning the experimental trials. Each subject then participated in four experimental trials that involved the ingestion of $600 \mathrm{ml}$ of distilled water to which glucose had been added to give concentrations of $0,2,5$ or $10 \%$. No electrolytes were added to the drinks. Measured osmolalities of 0 (SD 0) $\mathrm{mOsm} / \mathrm{kg}, 111$ (SD 1) $\mathrm{mOsm} / \mathrm{kg}, 266$ (SD 7) mOsm/kg and $565(\mathrm{sD} \mathrm{5)} \mathrm{mOsm} / \mathrm{kg}$ for the $0,2,5$ and $10 \%$ glucose solutions were, respectively, recorded. Experimental trials were separated by a period of at least $7 \mathrm{~d}$ and took place at the same time of the morning after an overnight fast. The subjects followed similar physical activity and nutritional intake patterns in the $24 \mathrm{~h}$ before each experimental trial. They were instructed to ingest $500 \mathrm{ml}$ of water approximately $1 \mathrm{~h}$ before arriving at the laboratory in an effort to ensure an adequate and consistent level of hydration status.

A urine sample was obtained upon arrival at the laboratory before body mass was measured using a beam balance (Marsdens type 150, Marsdens Weighing Machines, London, UK). A heart rate monitor was positioned (Polar Team System, Polar, Port Washington, NY, USA) before the subject sat upright in a room maintained at $22.4 \pm 0.5^{\circ} \mathrm{C}$ for a period of $10 \mathrm{~min}$. During this time, a $21 \mathrm{~g}$ cannula was inserted into a superficial forearm vein and it remained in place for the duration of the trial. The cannula was kept patent between sample collections by flushing with heparinised isotonic saline. Three $5 \mathrm{ml}$ blood samples were obtained at intervals of $10 \mathrm{~min}$. The subject was then given $5 \mathrm{~min}$ to consume $600 \mathrm{ml}$ of the test solution. All the drinks were warmed to a temperature of $37^{\circ} \mathrm{C}$ to avoid the peripheral vasoconstriction and consequent redistribution of blood flow that can occur after ingestion of cool drinks ${ }^{(15)}$. The blood samples $(5 \mathrm{ml})$ were obtained at $10 \mathrm{~min}$ intervals for $60 \mathrm{~min}$ following ingestion of the drink before a final urine sample was collected. The subjects remained seated for the duration of the trial to avoid the previously reported postural changes in plasma volume ${ }^{(16,17)}$.

\section{Sample analysis}

Blood samples were analysed for $\mathrm{Hb}$ concentration by the cyanmethaemoglobin method, packed-cell volume by microcentrifugation and glucose concentration by the glucose oxidase peroxidase amino-antipyrine phenol method (Randox, Crumlin, UK). The $\mathrm{Hb}$ and packed-cell volume values were used to estimate percentage changes in blood, erythrocyte and plasma volumes, as described by Dill \& Costill ${ }^{(18)}$.

An aliquot of whole blood was centrifuged at $1500 \mathrm{~g}$ for $15 \mathrm{~min}$ at $4^{\circ} \mathrm{C}$ before serum was removed and kept for the analysis of osmolality by freezing-point depression (Gonotec Osmomat 030 Cryoscopic Osmometer; Gonotec, Berlin, Germany) and sodium and potassium concentrations by flame photometry (Corning Clinical Flame Photometry 410C; Corning Ltd, Halstead, Essex, UK). Urine samples were analysed for osmolality using the method described previously.

All the analyses were performed in duplicate, with the exception of the packed-cell volume measurements, which were made in triplicate.

\section{Statistical analysis}

All datasets were tested for normal distribution using the Kolmogorov-Smirnov test. Parametric data are presented as means and standard deviations. Non-parametric data are presented as median (range) values.

Two-factor repeated-measures ANOVA were used to evaluate differences between the trials. A one-way ANOVA followed by Tukey's or Dunnett's pairwise comparisons was used for parametric post hoc tests. The Kruskal-Wallace test and Mann-Whitney pairwise comparisons were used as non-parametric post-tests. When appropriate, $t$ tests were used to locate the differences. The level of significance was taken as $P<0.05$, and this level of significance applies unless otherwise stated.

Statistical analysis was performed using SPSS 12.0 for windows.

\section{Results}

No difference in any of the measured blood parameters was observed between the three resting blood samples. Consequently, the third resting blood sample was used as a baseline value, as this was collected immediately before drinking. No difference in pre-ingestion blood parameters was observed between the trials.

\section{Change in blood, erythrocyte and plasma volume}

The two-factor repeated-measures ANOVA of the change in blood volume data showed no main effect of trial $(P=0.209)$, a main effect of time $(P<0.001)$ and an interaction between these factors $(P=0 \cdot 041)$. After ingestion of the $2 \%$ glucose solution, blood volume increased relative to the pre-ingestion value at 20 and $30 \mathrm{~min}$ and decreased at $60 \mathrm{~min}$ (Fig. 1). The two-factor repeated-measures ANOVA of the change in plasma volume data showed a main effect of trial $(P=0.014)$, a main effect of time $(P<0.001)$ and an interaction $(P=0.006)$. Plasma volume was elevated from baseline levels at $20 \mathrm{~min}$ after ingestion of the $2 \%$ glucose solution and decreased relative to the pre-ingestion value at 10 and $60 \mathrm{~min}$ after ingestion of the $10 \%$ glucose solution. At $10 \mathrm{~min}$ after ingestion of the test drinks, plasma volume 


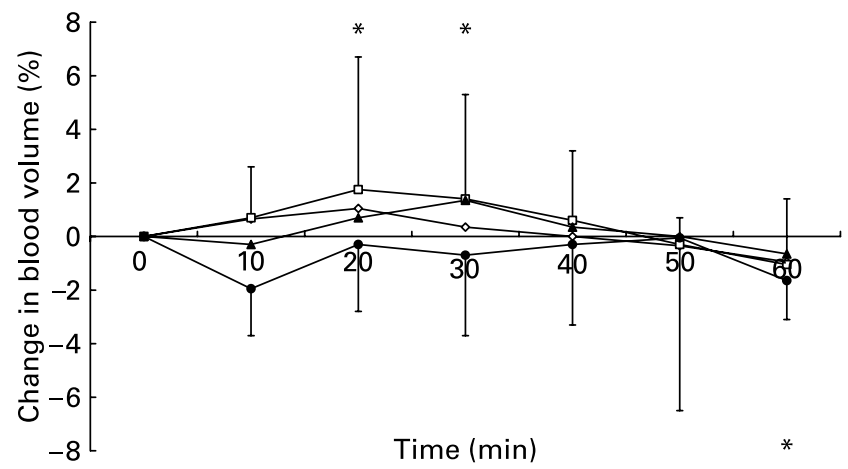

Fig. 1. Percentage change in blood volume following ingestion of the $0(-\diamond-), 2(-\square-), 5(-\mathbf{\Delta}-)$ and $10 \%(-\bullet-)$ glucose solutions. Time point 0 is the final pre-ingestion sample. Blood volume increased from baseline levels $\left({ }^{*} P<0.05\right)$ at 20 and $30 \mathrm{~min}$ and decreased from baseline levels $\left({ }^{\star} P<0.05\right)$ at $60 \mathrm{~min}$ after ingesting $2 \%$ glucose solution. The bars indicate median (range) values.

was higher during the 0 and $2 \%$ glucose trials than during the $10 \%$ glucose trial, and, at 20 and $30 \mathrm{~min}$ after ingestion, plasma volume remained higher during the $2 \%$ glucose trial than during the $10 \%$ glucose trial. At 30 and $40 \mathrm{~min}$ after ingestion, plasma volume was higher during the $5 \%$ glucose trial than during the $10 \%$ glucose trial (Fig. 2). No differences in erythrocyte volume were observed between the trials or over time.

\section{Serum osmolality}

Serum osmolality was higher during the 5 and $10 \%$ glucose trials than during the $0 \%$ glucose trial at $10 \mathrm{~min}$ after drink ingestion, and was also significantly higher during the $10 \%$ glucose trial than during the 0 and $2 \%$ glucose trials at 20 and $30 \mathrm{~min}$ after drink ingestion (Fig. 3). At $40 \mathrm{~min}$ after ingestion of the test solution, serum osmolality was higher during the $10 \%$ glucose trial than during the $0 \%$ glucose trial.

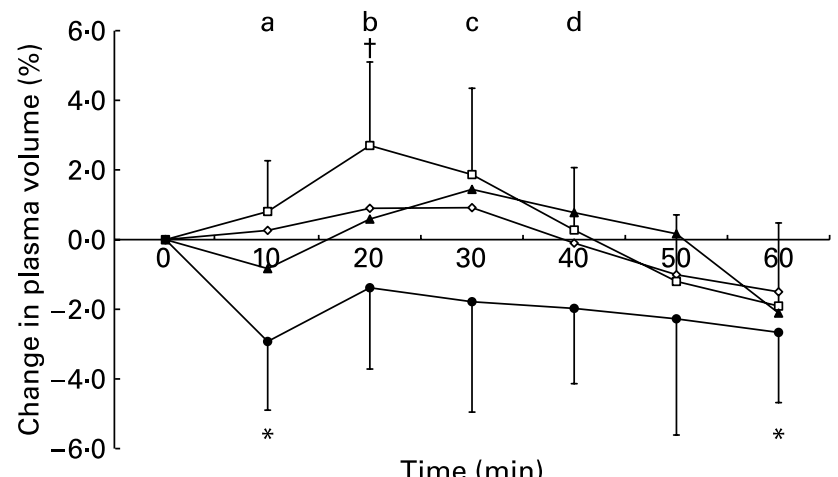

Fig. 2. Percentage change in plasma volume following ingestion of the $0(-\diamond-), 2(-\square-), 5(-\boldsymbol{\Delta}-)$ and $10 \%(-\bullet-)$ glucose solutions. Time point 0 is the final pre-ingestion sample. Plasma volume increased from baseline levels $(\dagger P<0.05)$ at $20 \mathrm{~min}$ after ingestion of the $2 \%$ glucose solution. Plasma volume decreased from baseline levels $\left({ }^{\star} P<0.05\right)$ at 10 and $60 \mathrm{~min}$ after ingestion of the $10 \%$ glucose solution. Plasma volume was higher during the following trials: (a) the 0 and $2 \%$ glucose trials compared with the $10 \%$ glucose trial at $10 \mathrm{~min}$; (b) the $2 \%$ glucose trial compared with the $10 \%$ glucose trial at $20 \mathrm{~min}$; (c) the 2 and $5 \%$ glucose trials compared with the $10 \%$ glucose trial at $30 \mathrm{~min}$; (d) the $5 \%$ glucose trial compared with the $10 \%$ glucose trial at $40 \mathrm{~min}$. Values are means and standard deviations.
Serum osmolality fell at $10 \mathrm{~min}$ after ingestion of the $0 \%$ glucose solution, but there were no significant deviations from baseline levels during the 2,5 or $10 \%$ glucose trials (Fig. 3).

\section{Heart rate}

Heart rate over the 30 min before drinking was rather stable and the average rate was taken as baseline. Heart rate was decreased from baseline levels at 30 and $40 \mathrm{~min}$ following ingestion of the $2 \%$ glucose solution (Fig. 4): this change amounted to about 3 beats/min from the baseline heart rate of about 64 beats $/ \mathrm{min}$. Heart rate was elevated during the drinking period on all trials. Heart rate was elevated from baseline levels at 10,50 and $60 \mathrm{~min}$ following ingestion of the $10 \%$ glucose solution and at $60 \mathrm{~min}$ following ingestion of the $5 \%$ glucose solution. Heart rate was lower during the $0 \%$ glucose trial than during the $10 \%$ glucose trial at 10 , $30,40,50$ and $60 \mathrm{~min}$ after ingestion and lower compared with the $5 \%$ glucose trial at $60 \mathrm{~min}$ after ingestion. During the $2 \%$ glucose trial, heart rate was lower than during the $10 \%$ glucose trial from 10 min after ingestion until the end of the experimental period, and was lower than during the $5 \%$ glucose trial at $10,20,30$ and 60 min after ingestion. A significant moderate negative correlation was observed between the percentage changes in plasma volume and heart rate (Pearson's correlation $=-0.445, P<0.001$ ).

\section{Blood glucose}

Blood glucose concentration (Fig. 5) was elevated from baseline levels at 10,20,30 and $40 \mathrm{~min}$ after ingestion of the $2 \%$ glucose solution and at all time points following ingestion of the 5 and $10 \%$ glucose solutions. Blood glucose concentration was lower during the $0 \%$ glucose trial than during all other trials at 10,20 and $30 \mathrm{~min}$ after ingestion and remained lower than during the 5 and $10 \%$ glucose trials until the end of the experimental period. Blood glucose concentration was lower during the $2 \%$ glucose trial than during the 5 and

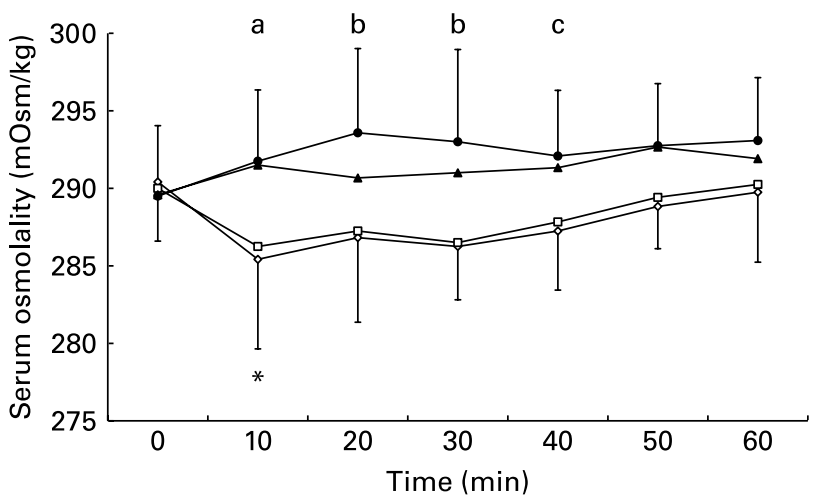

Fig. 3. Effect of ingestion of the $0(-\diamond-), 2(-\square-), 5(-\boldsymbol{\Delta}-)$ and $10 \%$ (-) glucose solutions on serum osmolality. Time point 0 is the final preingestion sample. Serum osmolality decreased from baseline levels ( $\left.{ }^{\star} P=0.029\right)$ at $10 \mathrm{~min}$ after ingestion of the $0 \%$ glucose solution. Serum osmolality was higher during the following trials: (a) the 5 and $10 \%$ glucose trials compared with the $0 \%$ glucose trial at $10 \mathrm{~min}$; (b) the $10 \%$ glucose trial compared with the 0 and $2 \%$ glucose trials at 20 and $30 \mathrm{~min}$; (c) the $10 \%$ glucose trial compared with the $0 \%$ glucose trial at $40 \mathrm{~min}$. 


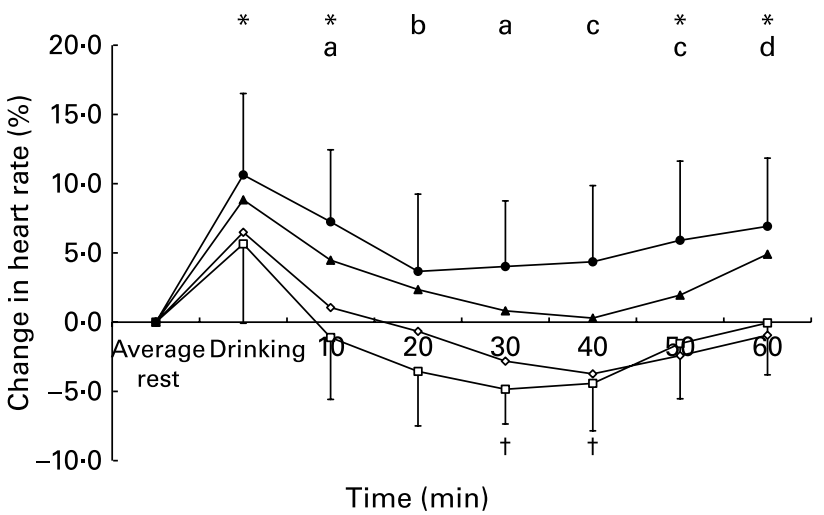

Fig. 4. Percentage change in heart rate following ingestion of the $0(-\diamond-)$, $2(-\square-), 5(-\Delta-)$ and $10 \%(-\bullet-)$ glucose solutions. Heart rate increased from baseline levels $\left({ }^{\star} P<0.05\right)$ during drinking of the $0,2,5$ and $10 \%$ glucose solutions at 10 and $50 \mathrm{~min}$ during the $10 \%$ glucose trial and at $60 \mathrm{~min}$ following ingestion of the 5 and $10 \%$ glucose solutions. Heart rate decreased from baseline levels $(\dagger P<0.05)$ at 30 and 40 min after ingestion of the $2 \%$ glucose solution. Heart rate was higher during the following trials: (a) the 5 and $10 \%$ glucose trials compared with the $2 \%$ glucose trial and the $10 \%$ glucose trial compared with the $0 \%$ glucose trial at 10 and $30 \mathrm{~min}$; (b) the 5 and $10 \%$ glucose trials compared with the $2 \%$ glucose trial at $20 \mathrm{~min}$; (c) the $10 \%$ glucose trial compared with the 0 and $2 \%$ glucose trials at 40 and $50 \mathrm{~min}$; (d) the 5 and $10 \%$ glucose trials compared with the 0 and $2 \%$ glucose trials at $60 \mathrm{~min}$. Values are means and standard deviations.

$10 \%$ glucose trials from 20 min after ingestion until the end of the experimental period.

\section{Serum electrolytes and urine measurements}

Serum sodium concentration was reduced from pre-ingestion values at $20 \mathrm{~min}$ after ingestion of the $0 \%$ glucose solution (Table 1). Table 2 shows urine volume and osmolality before and after ingestion of the drinks. Pre-trial urine volume was not different between the trials $(P=0.578)$ and the volume of urine passed at the end of the trials was not

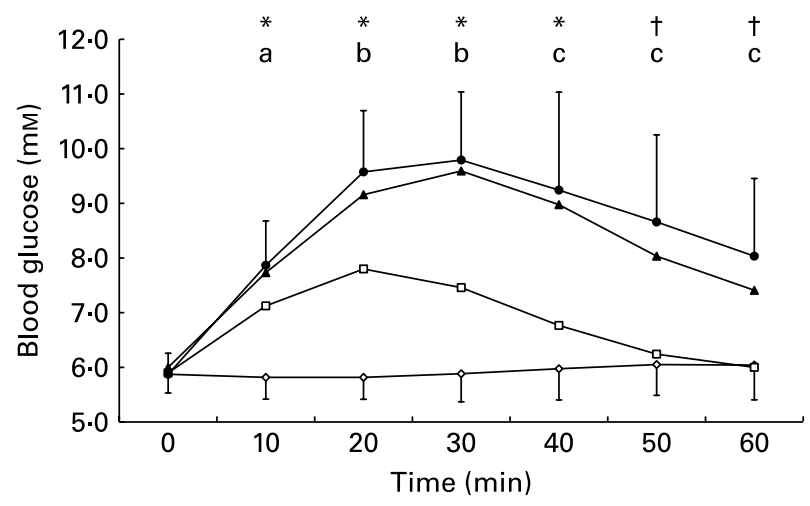

Fig. 5. Change in blood glucose concentration following ingestion of the 0 $(-\diamond-), 2(-\square-), 5(-\boldsymbol{\Delta}-)$ and $10 \%(-\bullet-)$ glucose solutions. Blood glucose concentration increased from baseline levels during the 2, 5 and $10 \%$ glucose trials at $10,20,30$ and $40 \mathrm{~min}(p<0.05)$ and was increased from baseline levels during the 5 and $10 \%$ glucose trials at 50 and $60 \mathrm{~min}(p<0.05)$. Blood glucose concentration was higher during the following trials: (a) the 2 , 5 and $10 \%$ glucose trials compared with the $0 \%$ glucose trial at $10 \mathrm{~min}$; (b) the 2,5 and $10 \%$ glucose trials compared with the $0 \%$ glucose trial and the 5 and $10 \%$ glucose trial compared with the $2 \%$ glucose trial at 20 and $30 \mathrm{~min}$; (c) the 5 and $10 \%$ glucose trials compared with the 0 and $2 \%$ glucose trials at 40,50 and $60 \mathrm{~min}$. Values are means and standard deviations. different between the trials $(P=0 \cdot 392)$. Pre-trial urine osmolality was not different between the trials $(P=0.968)$. Drinking significantly lowered urine osmolality, but there was no difference between the trials $(P=0 \cdot 465)$.

\section{Discussion}

The main finding of the present study is that ingestion of glucose solutions that are energy dense and have a high osmolality results in a reduction in plasma volume, while ingestion of a dilute $(2 \%)$ glucose solution results in an expansion of blood and plasma volume. These observations in intact healthy human subjects are consistent with the net movement of water between the small intestine and the extracellular fluid that occurs when hypotonic or hypertonic solutions are perfused directly into the small intestine ${ }^{(5)}$

Decreases in the water content of the blood which result in decreases in plasma volume and increases in serum osmolality lead to an increased viscosity of the blood. In turn, this results in an increased total peripheral resistance, decreased venous return, a decreased end-diastolic volume and a decreased stroke volume. It has been known since the early studies of Bainbridge that a decreasing venous return invokes a reflex increase in heart rate to maintain cardiac output ${ }^{(19)}$. Therefore, it is to be expected that a reduction in plasma volume as a result of the secretion of water drawn from the body pool into the gastrointestinal tract would result in an increase in the heart rate. In the present study, a significant moderate negative correlation was observed between the percentage changes in plasma volume and heart rate (Pearson's correlation $=-0.445, P<0.001)$. These small changes are unlikely to have any biological significance in healthy young individuals at rest, but may become important when there are constraints on the capacity to increase heart rate or to control blood pressure.

Studies of healthy older individuals have demonstrated a positive relationship between blood viscosity and arterial pressure $^{(13,14)}$. In hypertensive individuals, increases in blood viscosity, which the present study shows to occur following ingestion of strongly hypertonic solutions, may result in a further increase in peripheral resistance and contribute to an increased cardiac afterload that can result in left ventricular hypertrophy $^{(19)}$.

Table 1. Serum sodium concentrations (mM) following ingestion of the $0,2,5$ and $10 \%$ glucose solutions

(Mean values and standard deviations)

\begin{tabular}{|c|c|c|c|c|c|c|c|c|}
\hline & \multicolumn{8}{|c|}{ Glucose (\%) } \\
\hline & \multicolumn{2}{|c|}{0} & \multicolumn{2}{|c|}{2} & \multicolumn{2}{|c|}{5} & \multicolumn{2}{|c|}{10} \\
\hline & Mean & SD & Mean & SD & Mean & $\mathrm{SD}$ & Mean & SD \\
\hline Pre-ingestion & 139 & 2 & 139 & 3 & 139 & 2 & 139 & 3 \\
\hline 10 & 136 & 1 & 136 & 3 & 138 & 3 & 138 & 5 \\
\hline 20 & $136^{*}$ & 1 & 136 & 4 & 137 & 2 & 137 & 4 \\
\hline 30 & 137 & 2 & 135 & 4 & 137 & 3 & 137 & 5 \\
\hline 40 & 137 & 2 & 136 & 4 & 137 & 2 & 137 & 5 \\
\hline 50 & 138 & 3 & 137 & 4 & 138 & 3 & 137 & 5 \\
\hline 60 & 138 & 2 & 137 & 3 & 137 & 2 & 137 & 4 \\
\hline
\end{tabular}

* The mean value of the $0 \%$ glucose time point was significantly different from the pre-ingestion value. 
Table 2. Urine volume $(\mathrm{ml})$ and osmolality $(\mathrm{mOsm} / \mathrm{kg})$ before and $60 \mathrm{~min}$ after ingesting $0,2,5$ and $10 \%$ glucose solutions (Mean values and standard deviations)

\begin{tabular}{|c|c|c|c|c|c|c|c|c|}
\hline \multirow{3}{*}{$\begin{array}{l}\text { Glucose } \\
(\%)\end{array}$} & \multicolumn{4}{|c|}{ Urine volume } & \multicolumn{4}{|c|}{ Urine osmolality } \\
\hline & \multicolumn{2}{|c|}{ Pre-trial } & \multicolumn{2}{|c|}{ Post-trial } & \multicolumn{2}{|c|}{ Pre-trial } & \multicolumn{2}{|c|}{ Post-trial } \\
\hline & Mean & SD & Mean & SD & Mean & $\mathrm{SD}$ & Mean & SD \\
\hline 0 & 206 & 171 & 620 & 198 & 480 & 307 & 175 & 36 \\
\hline 2 & 265 & 163 & 493 & 196 & 473 & 327 & 191 & 48 \\
\hline 5 & 247 & 170 & 568 & 284 & 426 & 246 & 162 & 58 \\
\hline 10 & 181 & 132 & 482 & 198 & 465 & 267 & 189 & 59 \\
\hline
\end{tabular}

It may be inappropriate to make direct comparisons between results obtained from the intestinal perfusion model and oral ingestion studies ${ }^{(20)}$. The characteristics of a solution will change rapidly following oral ingestion due to mixing with gastric and intestinal secretions ${ }^{(21)}$. During perfusion studies, the test solution is constantly replaced, usually before full equilibration is achieved and water movement across only a small segment of the intestine can be monitored. The perfusion technique also looks only at a small part of the whole intestinal surface that is available for absorption in the intact individual; thus, concentrated solutions stimulate water secretion in the upper part of the intestine, but net absorption will normally occur in the distal regions. A previous study indicated that perfusion of the small intestine with a hypotonic (186 (SD 1) $\mathrm{mOsm} / \mathrm{kg}$ ) carbohydrate solution with small amounts of added electrolytes resulted in a $17 \%$ faster rate of water absorption than a hypertonic solution (403 (SD 3) $\mathrm{mOsm} / \mathrm{kg})^{(1)}$. In the present study, a $2 \%$ glucose solution with an osmolality of 111 (SD 1) $\mathrm{mOsm} / \mathrm{kg}$ resulted in significant increases in blood and plasma volume after ingestion. These results are consistent with a rapid rate of fluid absorption following ingestion of the dilute glucose solution.

It is well known that the activity of the intestinal sodium-glucose co-transporter is strongly influenced by the carbohydrate content of the diet ${ }^{(22)}$, and it seems likely that variations in the composition of the diet will account for part of the large inter-individual variability that is seen in the rates of gastric emptying and intestinal absorption. Although the transporter is upregulated in animals when carbohydrate is added to the diet, the response seems to be too slow to have any acute effect on the time scale of the present study, especially as the amount of glucose administered in the present study is small relative to the normal daily intake. Cheeseman ${ }^{(23)}$ showed that venous infusion of glucagon-like peptide- 2 resulted in rapid upregulation of sodium-glucose co-transporter in the rat intestine: a response was observed within $30 \mathrm{~min}$ of the onset of infusion and the effect was maximal at $1 \mathrm{~h}^{(23)}$. Perhaps more relevant are the observations of upregulation, within minutes, of the GLUT2 pathway in the presence of glucose, as reported by Kellett \& Helliwell $^{(24)}$ and subsequently confirmed in other publications. An excellent review was published by Kellett \& Brot-Laroche ${ }^{(25)}$. The rat, however, is not the same as the human, and it is not at present certain that such a rapid response would be observed in man after ingestion of a carbohydrate meal.

Leiper \& Maughan ${ }^{(5)}$ reported that perfusion of the jejunum with a hypertonic solution with an osmolality of 488 (SD 53) $\mathrm{mOsm} / \mathrm{kg}$ resulted in net water secretion into the small intestine. Ingestion of hypertonic solutions in the present study resulted in significant decreases in plasma volume, suggesting that ingestion of strongly hypertonic solutions results in a temporary net movement of water from the extracellular fluid into the intestinal lumen. This conclusion is supported by the serum osmolality data that were significantly higher during the $10 \%$ glucose trial than during the 0 and $2 \%$ glucose trials at a number of time points.

Strongly hypertonic solutions are ineffective rehydration beverages when rapid replacement of fluid losses is required, as rapid gastric emptying and intestinal absorption are essential for the maintenance of plasma volume ${ }^{(12)}$. By contrast, however, the challenge to rehydration after exercise-induced sweat losses is not to achieve rapid restoration of body water content, but rather to prevent the prompt loss of ingested fluid in urine when large volumes are consumed. Present advice suggests that, in order to properly rehydrate after exercise, an individual should drink a volume of fluid higher than that of body mass lost during exercise ${ }^{(26)}$. Ingestion of such large volumes of plain water or hypotonic carbohydrate-electrolyte drinks is likely to result in a rapid increase in blood and plasma volume, leading, in turn, to an acute decrease in plasma vasopressin and aldosterone concentrations and initiation of a diuretic response ${ }^{(27,28)}$. Ingestion of large volumes of strongly hypertonic carbohydrate solutions is likely to result in a decrease in plasma volume, causing plasma vasopressin and aldosterone concentrations to increase. This in turn is likely to lead to increased renal tubular reabsorption and a reduced renal water loss. There was no difference between the trials in urine output at the end of the $60 \mathrm{~min}$ study period. This is consistent with earlier studies where drinks with different electrolyte concentrations had no effect on urine output in the first hour after ingestion, but where a substantial reduction over the subsequent $3-5 \mathrm{~h}$ was observed when drinks with a high sodium content were ingested ${ }^{(29)}$.

Assuming that water accounts for about $70 \%$ of body mass in these subjects, extracellular fluid accounts for one-third of total body water and plasma volume constitutes one-quarter of the extracellular fluid ${ }^{(30)}$, the average reduction in plasma volume following ingestion of the highest osmolality solution in the present study would be approximately $96 \mathrm{ml}(2 \cdot 2 \%)$. During this trial, serum osmolality was about $5 \mathrm{mosmol} / \mathrm{kg}$ higher than during the 0 and $2 \%$ glucose trials, and blood glucose concentration increased by about $4 \mathrm{~mm}$, which would have resulted in an increased osmolality in the vascular space and a further redistribution of water among the various body compartments. This makes it difficult to estimate reductions in total extracellular fluid volume, but, if the reduction in total extracellular fluid was similar to the decrease in plasma 
volume, this equates to a decrease in extracellular fluid of approximately $384 \mathrm{ml}$. It would require approximately $700 \mathrm{ml}$ of free water to dilute the $10 \%$ glucose solution to isotonicity, but it is likely that a significant amount of hypertonic fluid remained in the gastrointestinal tract at the end of the $60 \mathrm{~min}$ measurement period ${ }^{(21,31)}$. It can, therefore, be concluded that the majority of water secreted into the small intestine following ingestion of hypertonic solutions is probably derived from the extracellular fluid.

In summary, the results of the present study suggest that ingestion of strongly hypertonic carbohydrate solutions results in a decrease in plasma volume, most likely, due to a temporary net secretion of water from the blood into the intestinal lumen. This may result in a transient hypohydration, but the resulting hypertonicity and a fall in plasma volume may prevent the initiation of the diuresis that accompanies ingestion of large volumes of dilute solutions.

\section{Acknowledgements}

The authors thank Dr Phillip Watson and Mr Orlando Laitano for their assistance in performing the present study. The study was not funded by any external body and the authors declare no conflict of interest in the conduction of the study. R. J. M. conceived and designed the study with assistance from S. M. S. Sample and data collection and analysis were undertaken by G. H. E. with assistance from S. M. S. All the authors contributed to the interpretation of the data and manuscript preparation.

\section{References}

1. Shi X, Summers RW, Schedl HP, et al. (1994) Effects of solution osmolality on absorption of select fluid replacement solutions in human duodenojejunum. J Appl Physiol 77, $1178-1184$.

2. Gisolfi CV, Summers RW, Schedl HP, et al. (1992) Intestinal water absorption from select carbohydrate solutions in humans. J Appl Physiol 73, 2142-2150.

3. Hunt JB, Thillainayagam AV, Salim AF, et al. (1992) Water and solute absorption from a new hypotonic oral rehydration solution: evaluation in human and animal perfusion models. Gut 33, 1652-1659.

4. Shi X, Summers RW, Schedl HP, et al. (1995) Effects of carbohydrate type and concentration and solution osmolality on water absorption. Med Sci Sports Exerc 27, 1607-1615.

5. Leiper JB \& Maughan RJ (1986) Absorption of water and electrolytes from hypotonic, isotonic and hypertonic solutions. J Physiol 373, 90P.

6. Lambert GP, Chang RT, Joensen D, et al. (1996) Simultaneous determination of gastric emptying and intestinal absorption during cycle exercise in humans. Int J Sports Med 17, 48-55.

7. Maughan RJ (1991) Fluid and electrolyte loss and replacement in exercise. In Perspectives in Exercise Science and Sports Medicine Volume 4: Ergogenics: The Enhancement of Sport Performance, pp. 35-85 [DR Lamb and MH Williams, editors]. Carmel, IN: Benchmark Press.

8. Leiper JB (2000) Gastric emptying and intestinal absorption of fluids, carbohydrates and electrolytes. In Sports Drinks, pp. 89-128 [RJ Maughan and R Murray, editors]. Boca Raton, FL: CRC Press.

9. Schedl HP \& Clifton JA (1963) Solute and water absorption by the human small intestine. Nature 199, 1264-1267.
10. Wapnir RA \& Lifshitz F (1985) Osmolality and solute concentration - their relationship with oral hydration solution effectiveness: an experimental assessment. Pediatr Res 19, 894-898.

11. Farthing MJG (2002) Oral rehydration: an evolving solution. $J$ Pediatr Gastroenterol Nutr 34, S64-S67.

12. Leiper JB (1998) Intestinal water absorption - implications for the formulation of rehydration solutions. Int J Sport Med 19, S129-S132.

13. Fowkes FGR, Lowe GDO, Rumley A, et al. (1993) The relationship between blood viscosity and blood pressure in a random sample of the population aged 55 to 74 years. Eur Heart J 14, 597-601.

14. Smith WC, Lowe GD, Lee AJ, et al. (1992) Rheological determinants of blood pressure in a Scottish adult population. J Hypertens 10, 467-472.

15. Imms FJ \& Lighten AD (1989) The cooling effects of a cold drink. In Thermal Physiology, pp. 135-139 [JB Mercer, editor]. New York: Elsevier Science.

16. Hagan RD, Diaz FJ \& Horvath SM (1978) Plasma volume changes with movement to supine and standing positions. J Appl Physiol 45, 414-418.

17. Shirreffs SM \& Maughan RJ (1994) The effect of posture change on blood volume, serum potassium and whole body electrical impedance. Eur J Appl Physiol 69, 461-463.

18. Dill DB \& Costill DL (1974) Calculation of percentage changes in volumes of blood, plasma, and red cells in dehydration. $J$ Appl Physiol. 37, 247-248.

19. Bainbridge FA (1915) The influence of venous filling upon the rate of the heart. J Physiol 50, 65-84

20. Schedl HP, Maughan RJ \& Gisolfi CV (1994) Intestinal absorption during rest and exercise: implications for formulating oral rehydration beverages. Med Sci Sports Exerc 26, 267-280.

21. Vist GE \& Maughan RJ (1995) The effect of osmolality and carbohydrate content on the rate of gastric emptying of liquids in man. $J$ Physiol 486, 523-531.

22. Ferraris RP (2001) Dietary and developmental regulation of intestinal sugar transport. Biochem J 360, 265-276.

23. Cheeseman CI (1997) Upregulation of SGLT-1 transport activity in rat jejunum induced by GLP-2 infusion in vivo. Am J Physiol 42, R1965-R1971.

24. Kellett GL \& Helliwell PA (2000) The diffusive component of intestinal glucose absorption is mediated by the glucose-induced recruitment of GLUT2 to the brush-border membrane. Biochem $J$ 350, 155-162.

25. Kellett GL \& Brot-Laroche E (2005) Apical GLUT2. A major pathway of intestinal sugar absorption. Diabetes 54, 3056-3062.

26. Shirreffs SM, Taylor AJ, Leiper JB, et al. (1996) Post-exercise rehydration in man: effects of volume consumed and drink sodium content. Med Sci Sports Exerc 28, 1260-1271.

27. Kenefick RW, Maresh CM, Armstrong LE, et al. (2000) Plasma vasopressin and aldosterone responses to oral and intravenous saline rehydration. J Appl Physiol 89, 2117-2122.

28. Melin B, Jimenez C, Savourey G, et al. (1997) Effects of hydration state on hormonal and renal responses during moderate exercise in the heat. Eur J Appl Physiol 76, 320-327.

29. Shirreffs SM \& Maughan RJ (1998) Volume repletion following exercise-induced volume depletion in man: replacement of water and sodium losses. Am J Physiol 43, F868-F875.

30. Sawka MN (1990) Body fluid responses and hypohydration during exercise-heat stress. In Human Performance Physiology and Environmental Medicine at Terrestrial Extremes, pp. 227-266 [KB Pandolf, MN Sawka and RR Gonzalez, editors]. Carmel, IN: Cooper Publishing Group.

31. Vist GE \& Maughan RJ (1994) The effect of increasing glucose concentration on the rate of gastric emptying in man. Med Sci Sports Exerc 26, 1269-1273. 